\title{
NEW EXAMPLES OF COMPLETE RICCI SOLITONS
}

\author{
THOMAS IVEY
}

(Communicated by Peter Li)

\begin{abstract}
The Ricci soliton condition reduces to a set of ODEs when one assumes that the metric is a doubly-warped product of a ray with a sphere and an Einstein manifold. If the Einstein manifold has positive Ricci curvature, we show there is a one-parameter family of solutions which give complete noncompact Ricci solitons.
\end{abstract}

\section{INTRODUCTION}

A Ricci soliton is a solution to the Ricci flow $\partial g / \partial t=-2 \operatorname{Ric}(g)$ such that the metric changes only by diffeomorphisms as time goes on; since the diffeomorphisms of the underlying manifold are symmetries of the evolution equation, it would be more accurate to call this a similarity solution for the Ricci flow. Soliton solutions are important to the study of the Ricci flow because they represent extremal cases for the Harnack estimate [H2] and may be limiting cases for the Ricci flow near singularities (cf. [A]).

A Ricci soliton is generated by an initial metric $g$ and a vector field $V$ such that $\mathscr{L}_{V} g=2 \operatorname{Ric}(g)$; then $V$ generates the diffeomorphisms. A gradient soliton is one where $V$ is the gradient of some function $h$ with respect to $g$; the corresponding condition is that the Hessian $\nabla^{2} h$ coincide with the Ricci tensor. Up to now, the known examples of complete Ricci solitons were the radially symmetric 'cigar' metric on $\mathbb{R}^{2}[\mathrm{H} 1]$, the radially symmetric soliton on $\mathbb{R}^{3}$ discovered by Bryant (which easily generalizes to $\mathbb{R}^{n}$ ), and the $U(n)$ symmetric soliton on $\mathbb{C}^{n}$ disovered by Cao [C].

Let $\left(M^{n}, d \sigma^{2}\right)$ be a compact Einstein manifold with Einstein constant $\epsilon>$ 0 . Let $d \theta^{2}$ denote the standard metric of constant curvature +1 on $S^{k}, k \geq 1$. On $\mathbb{R}^{k+1} \times M$ with radial coordinate $t \geq 0$ consider the doubly-warped product metric

$$
d s^{2}=d t^{2}+f(t)^{2} d \theta^{2}+g(t)^{2} d \sigma^{2} .
$$

For the metric to be smooth near $t=0$ we require that $f$ extend smoothly to

Received by the editors December 2, 1992.

1991 Mathematics Subject Classification. Primary 53C25; Secondary 34C99.

Key words and phrases. Ricci solitons.

Research supported by a fellowship from the Natural Sciences and Engineering Research Council of Canada and a fellowship from Duke University. 
an odd function of $t$ with $f^{\prime}(0)=1$ and $g$ extend smoothly to a positive even function of $t$ (cf. [BB, §8.9]).

Theorem. There exists a one-parameter family of smooth complete Ricci soliton metrics of the form (*) on $\mathbb{R}^{k+1} \times M$. These metrics have positive Ricci curvature for $t>0$ and positive scalar curvature everywhere. For a given $M$, no two of these metrics are equivalent under diffeomorphisms of $\mathbb{R}^{k+1} \times M$ that preserve the submersion onto $\mathbb{R}^{k+1}$.

\section{THE CONSTRUCTION}

We will construct gradient solitons where $V$ is the gradient of a function $h$ of $t$ only; $V$ will be smooth if $h^{\prime}$ extends to a smooth odd function of $t$. Setting $\nabla^{2} h$ equal to the Ricci tensor gives the following system of ODEs:

$$
\left\{\begin{array}{l}
h^{\prime \prime}=-n \frac{g^{\prime \prime}}{g}-k \frac{f^{\prime \prime}}{f}, \\
\frac{f^{\prime \prime}}{f}=-n \frac{f^{\prime} g^{\prime}}{f g}-\frac{h^{\prime} f^{\prime}}{f}+(k-1)\left(\frac{1-\left(f^{\prime}\right)^{2}}{f^{2}}\right), \\
\frac{g^{\prime \prime}}{g}=\frac{\epsilon}{g^{2}}-(n-1)\left(\frac{g^{\prime}}{g}\right)^{2}-k \frac{f^{\prime} g^{\prime}}{f g}-h^{\prime} \frac{g^{\prime}}{g} .
\end{array}\right.
$$

(From now on we will assume $\epsilon=1$.) Given a solution of these equations defined for $t>0$, we only need to check that the one-sided limits of $f, g$, $f^{\prime}$, and $h^{\prime}$ as $t \rightarrow 0$ are as required, and that the right-hand sides of the ODEs have finite limits. Then the odd, even, and odd extensions of $f, g$, and $h^{\prime}$, respectively, are smooth solutions to (1) for all $t$; for more details, see [I].

Two features of (1) simplify the analysis of the solutions. First of all, there is a first integral which arises from the Bianchi identity: if we substitute $\nabla^{2} h=$ Ric into the formula $g^{i k}\left(h_{i j k}-h_{i k j}\right)=h_{p} R^{p}{ }_{j}$, and use the second Bianchi identity $2 g^{i j} \nabla_{j} R_{i k}=\nabla_{k} R$ for the Ricci tensor, we get $d\left(\Delta h+\left(h^{\prime}\right)^{2}\right)=0$. (By the Laplacian we mean the trace of the Hessian.) Next, we will reduce the number of variables in (1) by rewriting the system in terms of quantities invariant under the symmetries of (1), which consist of scaling simultaneously in $f, g$, and $t$ and, in the case $k=1$, scaling $f$ by itself. The quantities we will use are

$$
\begin{aligned}
X & =g^{\prime} Y, & Y & =\sqrt{n}\left(g h^{\prime}+n g^{\prime}+\frac{k f^{\prime} g}{f}\right)^{-1}, \\
Z & =\frac{\sqrt{k}}{\sqrt{n}} \frac{f^{\prime} g}{f} Y, & W & =\frac{\sqrt{k(k-1)}}{\sqrt{n}} \frac{g}{f} Y .
\end{aligned}
$$

With this choice, the first integral becomes

$$
X^{2}+Y^{2}+Z^{2}+W^{2}=1+C g^{2} Y^{2},
$$

where $C$ is an arbitrary constant. Since $g$ is not invariant under the symmetries of (1) but appears as a common factor in the $t$-derivatives of $X$, $Y, Z$, and $W$, we get rid of it by defining a new parameter $s$ such that 
$\sqrt{n} d t=g Y d s ;$ then

$$
\left\{\begin{array}{l}
\frac{d X}{d s}=X\left(X^{2}+Z^{2}-1\right)+\alpha Y^{2}, \\
\frac{d Y}{d s}=Y\left(X^{2}+Z^{2}-\alpha X\right), \\
\frac{d Z}{d s}=Z\left(X^{2}+Z^{2}-1\right)+\beta W^{2}, \\
\frac{d W}{d s}=W\left(X^{2}+Z^{2}-\beta Z\right),
\end{array}\right.
$$

where $\alpha=1 / \sqrt{n}$ and $\beta=1 / \sqrt{k}$. (When $k=1, g / f$ is not invariant under the extra symmetry; but then $W$ is zero anyway, and we obtain the restriction of the above system to the hyperplane $W=0$.)

It is clear from the first integral that the unit sphere in $X Y Z W$ space is stable under (3). In fact, there is a Lyapunov function:

$$
\frac{d}{d s}\left(X^{2}+Y^{2}+Z^{2}+W^{2}-1\right)=2\left(X^{2}+Y^{2}+Z^{2}+W^{2}-1\right)\left(X^{2}+Z^{2}\right) .
$$

From (2), the required limits for $f, g, f^{\prime}$, and $h$ as $t \rightarrow 0$ imply that we should have $X \rightarrow 0, Y \rightarrow 0, Z \rightarrow \beta$, and $W \rightarrow \sqrt{1-\beta^{2}}$. This is a critical point of (3) on the unit sphere; linearization of (3) about this point shows that the Jacobian has two real positive eigenvalues. Thus the unstable manifold of this point is a surface, and in it there is a one-parameter family of integral curves of (3) that tend exponentially to this point as $s \rightarrow-\infty$ (cf. the Central Manifold Theorem in [AA]). We will restrict our attention to integral curves in the unstable manifold which lie inside the open ball with boundary the unit sphere. By (4), these curves approach the origin in $X Y Z W$ space exponentially as $s \rightarrow+\infty$.

\section{SKETCH OF THEOREM}

Given one of these curves, we can recover $f, g, h^{\prime}$, and $t$ by quadrature:

$$
\begin{aligned}
\frac{d g}{g}=\frac{X(Y d X-Y d X)}{Y\left(X^{2}-\sqrt{n} X+Y^{2}\right)}, & \frac{d t}{g}=\frac{Y d X-X d Y}{X^{2}-\sqrt{n} X+Y^{2}}, \\
\frac{d f}{f}=\frac{Z(Y d X-X d Y)}{\alpha Y\left(X^{2}-\sqrt{n} X+Y^{2}\right)}, & g h^{\prime}=\frac{\sqrt{n}-X-\sqrt{n / k} Z}{Y} .
\end{aligned}
$$

(When $k>1$ we can use the shortcut $f=\frac{k(k-1)}{\sqrt{n}} \frac{g Y}{W}$.) Showing that one can get $g$ to approach a positive limit and $t$ to approach zero as $s \rightarrow-\infty$ comes down to showing that the limiting value of $X / Y^{2}$ as the curve approaches $\left(0,0, \beta, \sqrt{1-\beta^{2}}\right)$ is $\alpha /\left(1+\beta^{2}\right)$. (Clearly the integral curves of (3) will be symmetric about the $Y=0$ hyperplane; this is the value one gets from a naive Taylor series expansion for $X$ as a function of $Y^{2}$.) The equations

$$
\begin{gathered}
\frac{d X}{d s}=X\left(X^{2}+Z^{2}-1\right)+\alpha Y^{2} \\
\frac{d}{d s}\left(\left(1+\beta^{2}\right) X-\alpha Y^{2}\right)=\left(X^{2}+Z^{2}-1\right)\left(\left(1+\beta^{2}\right) X-\alpha Y^{2}\right)+\alpha Y\left(Y\left(1+\beta^{2}\right)+\alpha X-1\right)
\end{gathered}
$$


show that $0 \leq X / Y^{2} \leq \alpha /\left(1+\beta^{2}\right)$ for $X$ and $Y$ near zero. Once we know $X / Y^{2}$ is bounded in absolute value,

$$
2 \frac{d / d s X}{d / d s Y^{2}}=\frac{X}{Y^{2}}\left(\frac{X^{2}+Z^{2}-1}{X^{2}+Z^{2}-\alpha X}\right)+\frac{\alpha}{X^{2}+Z^{2}-\alpha X}
$$

shows that $X / Y^{2}$ has the limiting value $\alpha /\left(1+\beta^{2}\right) .^{1}$ In a similar way one argues that $\bar{Z}=(Z-\beta) / Y^{2}$ and $\bar{W}=\left(W-\sqrt{1-\beta^{2}}\right) / Y^{2}$ have finite limits as $s \rightarrow-\infty$, and this lets us verify the condition on the right-hand sides of (1) near $t=0$. (In fact, using $\bar{Z}$ and $\bar{W}$ as blowup coordinates near the critical point shows that the limit of $\bar{Z}$ determines that of $\bar{W}$ and uniquely determines the integral curve.)

It remains to check that the metrics produced by these integral curves are complete. First one shows that for any curves coming into the origin from the region where $X$ and $Y$ are positive, the limit of $X / Y^{2}$ is $\alpha$. Furthermore, the limit of $\left(X-\alpha Y^{2}\right) / Y^{4}$ is $2 \alpha^{3}$; this calculation also involves showing $Z / Y^{2}$ is bounded near the origin. Finally, one uses the above quadratures to show that $g$ is asymptotic to $1 / Y$ and $\int d t$ tends to infinity as $Y \rightarrow 0$.

To check the sign of the Ricci curvature, it is easiest to use the Hessian

$$
\nabla^{2} h=h^{\prime \prime} d t^{2}+f f^{\prime} h^{\prime} d \theta^{2}+g g^{\prime} h^{\prime} d \sigma^{2} .
$$

Checking that $h^{\prime}>0$ and $h^{\prime \prime}>0$ for $t>0$ is equivalent to checking that $1-\sqrt{n} X-\sqrt{k} Z>0$ and $\sqrt{n} X+\sqrt{k} Z-X^{2}-Y^{2}-Z^{2}-W^{2}>0$ on the integral curves; this is easily done by computing the $s$-derivatives. Moreover, the first integral $\Delta h+\left(h^{\prime}\right)^{2}=C$ shows that the scalar curvature $R=\Delta h$ must be positive for $t=0$, and by symmetry all the eigenvalues of the Ricci curvature along $\mathbb{R}^{k+1}$ must be positive there. However, the eigenvalues along the fibre $M$ have limit zero as $t \rightarrow 0$, so this prevents us from having strictly positive Ricci curvature.

To verify the third claim in the theorem, it is sufficient to show that from the metric one can recover the integral curve of (3). This can be done as follows: the origin in $\mathbb{R}^{k+1}$ is the point over which the fibres of the submersion have least volume; $t$ is the distance in $\mathbb{R}^{k+1}$ to this point; the Einstein constant in the fibres is $1 / g(t)$, and the volume of the distance spheres in $\mathbb{R}^{k+1}$ is $f(t)$, up to a universal constant depending on $k$. Finally, when Ric $>0$ and the vector field $V$ is the gradient of a function $h$, the identity $d R=-2 \operatorname{Ric}(V$,$) , which$ arises from commuting two covariant derivatives of $V$, uniquely determines $V$.

\section{REMARKS}

The previously known examples of complete Ricci solitons have strictly positive curvature operator. (This means the curvature tensor is positive definite when considered as a quadratic form on the second exterior power of the tangent bundle, and it implies positive sectional curvature.) This is as far as the 'technology' for the Ricci flow has gone; the most significant results, such as convergence for the Ricci flow in the noncompact case [S] and the Harnack estimate, have been obtained under the assumption of positive sectional curvature

\footnotetext{
${ }^{1}$ This follows from a recursive form of l'Hôpital's Rule: if $f^{\prime}(x) / g^{\prime}(x)=q(x) f(x) / g(x)$ $+p(x), f / g$ is bounded as $x \rightarrow a$, and $\lim q(x)$ is finite and different from 1 , then $\lim f / g=$ $\lim (p /(1-q))$.
} 
and positive curvature operator, respectively. Indeed, the most general curvature condition known to be preserved by the Ricci flow is nonnegative curvature operator.

In our construction, $M$ can be any Einstein manifold with positive Ricci curvature, so we do not have much control over the sectional curvature along the copies of $M$. In fact, if $\pi$ is a two-plane tangent to one of the fibres $M$ and $K_{M}(\pi)$ is its sectional curvature under the Einstein metric, then its sectional curvature under the soliton metric is

$$
K(\pi)=\frac{K_{M}(\pi)-\left(g^{\prime}\right)^{2}}{g^{2}} .
$$

In our construction, $g^{\prime}$ is not identically zero, so if $K_{M}(\pi)=0$ we get negative sectional curvature on some fibres. The lowest dimension when this can happen is when the dimension of $M$ is four: we can use $M=S^{2} \times S^{2}$ and $k=1$ to produce a six-dimensional complete Ricci soliton with sectional curvature of mixed sign.

\section{ACKNOWLEDGMENT}

The author would like to thank Robert Bryant for helpful discussions and encouragement while this work was carried out.

\section{REFERENCES}

[A] S. Angenent, On the formation of singularities in the curve shortening flow, J. Differential Geom. 33 (1991) 601-633.

[AA] D. V. Anosov and V. I. Arnold (eds.), Encyclopaedia of mathematical sciences, Vol. I: Dynamical systems, Springer-Verlag, New York, 1988.

[BB] L. Bérard-Bergery, Sur de nouvelles variétiés riemannienes d'Einstein, Publ. Inst. E. Cartan, vol. 4, Univ. de Nancy, 1982.

[C] H. D. Cao, On Harnack's inequalities for the Kähler-Ricci flow, Invent. Math. 109 (1992), 247-263.

[H1] R. Hamilton, The Ricci flow on surfaces, Contemp. Math., vol. 71, Amer. Math. Soc., Providence, RI, 1988, pp. 237-262.

[H2] _ , The Harnack estimate for the Ricci flow, J. Differential Geom. 37 (1993), 225-243.

[I] T. Ivey, On solitons for the Ricci flow, Ph.D. Thesis, Duke Univ., 1992.

[S] W.-X. Shi, Ricci deformation of the metric on complete noncompact Riemannian manifolds, J. Differential Geom. 30 (1989).

Department of Mathematics, University of California at San Diego, la Jolla, CaliFORNIA 92093-0112

E-mail address: tivey@euclid.ucsd.edu 